\title{
SHORT STORIES
}

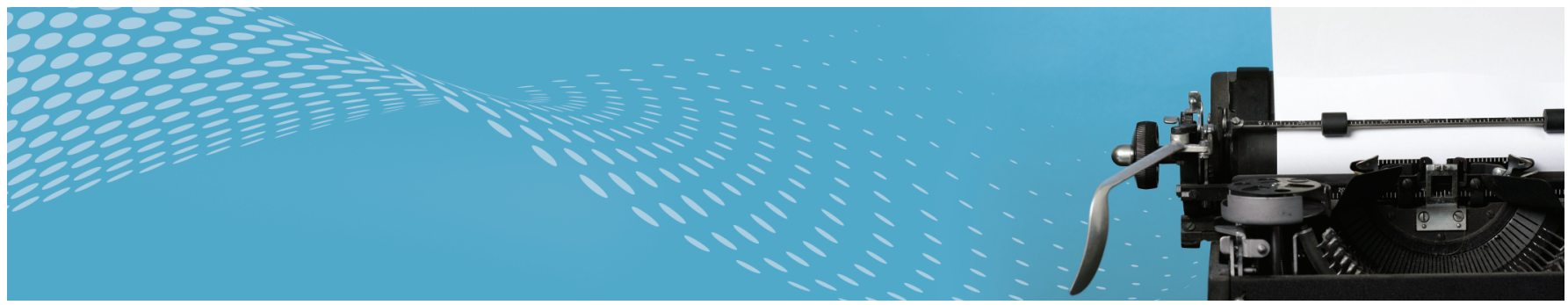

\section{Pentagonum Pentagonorum}

\section{Danny Calegari}

Pentagon of pentagons, all is pentagon. So begins Ecclesiastes, a hymn to the pointlessness of human activity, and my favorite book of the Old Testament.

An $n$-gon in the Euclidean plane is determined by $2 n$ parameters: the locations of the vertices. Dividing by the group of similarities reduces this to $2 n-4$ parameters; prescribing the interior angles imposes a further $n-1$ conditions (because the angle sum is constant) reducing this to $n-3$. An equiangular Euclidean pentagon $P$ is one whose interior angles are all equal (to $3 \pi / 5$ ); see Figure 1 .

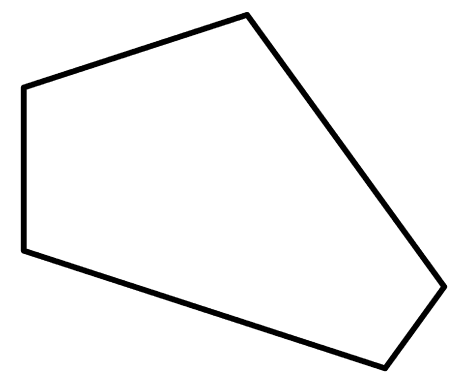

Figure 1. An equiangular Euclidean pentagon $P$.

If we denote $\zeta=e^{2 \pi i / 5}$, the successive edge lengths $L_{j}$ satisfy

$$
\sum_{j=0}^{4} L_{j} \zeta^{j}=0
$$

Danny Calegari is a professor of mathematics at the University of Chicago. His email address is dannyc@math.uchicago.edu.

For permission to reprint this article, please contact:

reprint-permission@ams.org.

DOI: https://doi.org/10.1090/noti2331 which can be thought of as a pair of real linear equalities that cut out a real three-dimensional space $L$ in $\mathbb{R}^{5}$ with coordinates $L_{j}$. The projectivization $\mathbb{P} L$ is a real projective plane, and within this plane the geometric inequalities $L_{j}>0$ cut out the interior of a pentagon $\Pi \subset \mathbb{P} L-$ the parameter space ${ }^{1}$ of equiangular pentagons; see Figure 2. The boundary of $\Pi$ parameterizes "degenerate" equiangular pentagons: the edges, resp., vertices, correspond to equiangular "pentagons" with one, resp., two (nonadjacent), edge lengths going to zero. Notice that adjacent edges of $\Pi$ correspond to degenerations of nonadjacent sides of $P$.

The area $A(P)$ of a polygon $P$ is a quadratic form on $L$ with signature $(2,1)$. Note that when some edge lengths are negative the associated "polygon" might be immersed, and we must measure area in the algebraic sense; thus $A(P)$ can be positive, negative, or zero. The quadratic form $A$ vanishes on a cone in $L$ that projects to a conic $C$ in $\mathbb{P} L$ (i.e., a circle), dividing it into a disk region and a Möbius band. "Honest" polygons-those with all $L_{i}$ positivehave positive area, so $\Pi$ is contained on the disk side of $C$. The group of projective automorphisms of $\mathbb{P} L$ fixing $C$ preserves a metric in the disk-the so-called Klein model of the hyperbolic plane. Projectively straight lines become geodesics in this metric, so $\Pi$ is a hyperbolic pentagon. By symmetry, the interior angles of $\Pi$ (in the hyperbolic metric) are all equal; in fact, we shall see that these angles are all equal to $\pi / 2$, i.e., $\Pi$ is a regular hyperbolic right-angled pentagon.

\footnotetext{
${ }^{1}$ Technically these pentagons are "marked" by a distinguished vertex, or else we would have to quotient out by a further order 5 symmetry that cyclically permutes the $L_{j}$, but we ignore this point.
} 


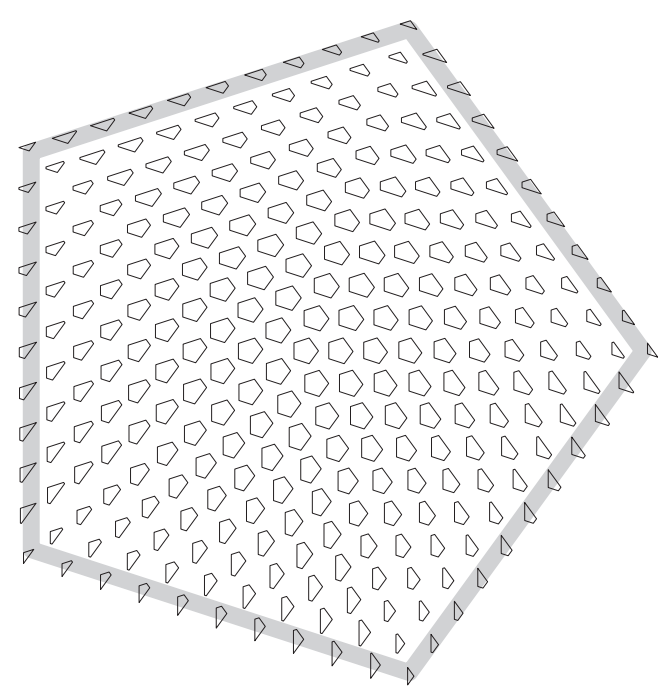

Figure 2. The pentagon of pentagons $\Pi$.

For each index $j$ there is a linear involution $\iota_{j}$ on $L$ that takes $L_{j}$ to $-L_{j}$, and takes $L_{j \pm 1}$ to $L_{j \pm 1}+2 \phi L_{j}$, where $\phi:=1 /\left(\zeta+\zeta^{-1}\right)$ is the Golden Ratio. This involution amounts to adding two times the fundamental solution $(\phi,-1, \phi, 0,0)$ to equation (1) or a cyclic conjugate. Observe that $\iota_{j}$ and $\iota_{j^{\prime}}$ commute whenever $\left|j-j^{\prime}\right| \neq 1$. Geometrically, this operation corresponds to adding on or cutting off a "bowtie" $\bowtie$ along an edge of $P$, so we refer to it as a bowtie move; see Figure 3.
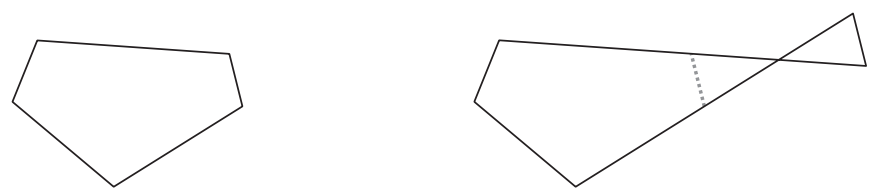

Figure 3. A bowtie move on $P$ is reflection in a side of $\Pi$.

A bowtie move creates (or destroys) two similar triangles with opposite orientation, that enclose regions whose algebraic areas cancel. Thus the bowtie move leaves $A$ fixed, and therefore each $\iota_{j}$ projects to an isometry of the hyperbolic plane of order two: a hyperbolic reflection. The involution $\iota_{j}$ fixes the edge of $\Pi$ parameterizing degenerate polygons with $L_{j}=0$, so we see that the $\iota_{j}$ are simply the hyperbolic reflections in the edges of $\Pi$, and the fact that $\iota_{j}$ and $\iota_{j^{\prime}}$ commute when $\left|j-j^{\prime}\right| \neq 1$ implies that adjacent edges of $\Pi$ are perpendicular. This completes the proof that $\Pi$ is a regular right-angled hyperbolic pentagon.

By the way, the group generated by the $l_{j}$ is an example of a right-angled Coxeter group: a group generated by involutions, with relations saying that some subset of pairs of generators commute. Right-angled Coxeter groups are usually encoded by graphs (called diagrams), with one vertex for each generator and an edge between two vertices if the associated generators commute; another convention is that there is an edge between two vertices if the associated generators don't commute. Fortunately, in this example, the diagram of the Coxeter group with either convention is the same, and is ... well, it's indicated in Figure 4.

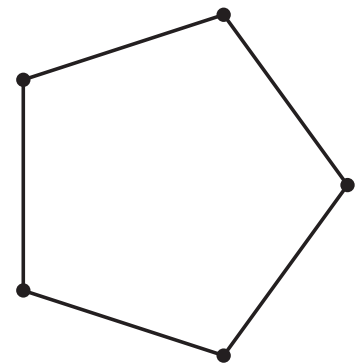

Figure 4. Coxeter diagram of the group generated by reflections in sides of $\Pi$.

If the regular right-angled hyperbolic pentagon parameterizes equiangular Euclidean pentagons, what do the other right-angled hyperbolic pentagons parameterize? Let $\zeta \in \mathbb{C}^{5}$ have coordinates $\zeta_{j}$, not too far from $\zeta^{j}$, and let's let $L(\zeta) \subset \mathbb{R}^{5}$ denote the real vector space of solutions to $\sum_{j=0}^{4} L_{j} \zeta_{j}=0$. A vector in $L(\zeta)$ with all coefficients positive determines a convex Euclidean pentagon with consecutive oriented edges parallel to the $\zeta_{j}$. The area of this pentagon defines a quadratic form $A(\zeta)$ on $L(\zeta)$, and the projectivization of the orthant $L_{j} \geq 0$ is a hyperbolic pentagon $\Pi(\zeta)$ in the disk bounded by the projectivization of the cone $A(\zeta)=0$. Bowtie moves make sense as operations on pentagons in $L(\zeta)$, and induce reflections in the edges of $\Pi(\zeta)$. Since bowtie moves on nonadjacent sides commute, $\Pi(\zeta)$ is right-angled. All isometry types of right-angled hyperbolic pentagons arise from suitable $\zeta$.

How can we describe the parameter space $\mathcal{P}$ of rightangled hyperbolic pentagons this way? There are two obvious kinds of transformations of $\zeta$ which leave the geometry of $\Pi(\zeta)$ unchanged. First, we can scale the $\zeta_{j}$ by any positive real numbers $t_{j}$. If we define $\omega_{j}:=t_{j} \zeta_{j}$, then the linear endomorphism of $\mathbb{R}^{5}$ that scales $L_{j}$ by $1 / t_{j}$ takes $L(\zeta)$ to $L(\omega)$, preserves the positive orthant, and takes the quadratic form $A(\zeta)$ to $A(\omega)$. It follows that the pentagons $\Pi(\zeta)$ and $\Pi(\omega)$ are isometric in their respective hyperbolic spaces. Second, we can identify $\mathbb{C}$ with $\mathbb{R}^{2}$ and act simultaneously on the elements of $\zeta$ (which we think of as vectors in $\mathbb{R}^{2}$ ) by a linear transformation $T \in \operatorname{SL}(2, \mathbb{R})$. Then $L(\zeta)=L(T(\zeta))$ as vector spaces, and since $T$ acts on Euclidean polygons in an area-preserving way, the quadratic forms $A(\zeta)$ and $A(T(\zeta))$ agree. Thus we only need to consider the arguments of the $\zeta_{j}$, up to the projective action of $\operatorname{SL}(2, \mathbb{R})$ on the unit circle (note this is the double-cover of the "usual" projective action of $\operatorname{PSL}(2, \mathbb{R})$ on $\left.\mathbb{R P}^{1}\right)$.

In order for positive $L$ in $L(\zeta)$ to parameterize genuine convex Euclidean pentagons, the arguments of the $\zeta_{j}$ should appear in the "correct" cyclic order, and each 
$-\zeta_{j}$ should be a positive real linear combination of $\zeta_{j-2}$ and $\zeta_{j+2}$ (so that the degeneration $L_{j-1}=L_{j+1}=0$ corresponds to a degeneration of an oriented convex pentagon to a triangle). Equivalently, the images of the $\zeta_{j}$ in the projective line $\mathbb{R} \mathbb{P}^{1}$ should appear in the cyclic order $0,3,1,4,2$; i.e., they should be the vertices of a pentagram.

Let's relabel the images of $\zeta_{0}, \zeta_{3}, \zeta_{1}, \zeta_{4}, \zeta_{2}$ in $\mathbb{R} \mathbb{P}^{1}$ in the cyclic order they appear, as $p_{0}, p_{1}, p_{2}, p_{3}, p_{4} \in \mathbb{R P}^{1}$. A complete projective invariant of four points $p_{0}, p_{1}, p_{2}, p_{3}$ in $\mathbb{R P}^{1}$ is the cross ratio

$$
\left[p_{0}, p_{1} ; p_{2}, p_{3}\right]:=\frac{\left(p_{2}-p_{0}\right)\left(p_{3}-p_{1}\right)}{\left(p_{2}-p_{1}\right)\left(p_{3}-p_{0}\right)} .
$$

Five points $p_{0}, p_{1}, p_{2}, p_{3}, p_{4}$ give rise to five cross ratios $X_{j}:=\left[p_{j}, p_{j+1} ; p_{j+2}, p_{j+3}\right]$, indices taken mod 5 . These satisfy identities

$$
\left(X_{j+2}-1\right)\left(X_{j-2}-1\right)=\frac{\left(X_{j}-1\right)}{X_{j}} .
$$

The condition that the $p_{j}$ appear in cyclic order is equivalent to inequalities for successive cross ratios:

$$
1<X_{j+1}<\frac{X_{j}}{X_{j}-1} .
$$

By trigonometry, the successive lengths $\ell_{j}$ of a rightangled hyperbolic pentagon satisfy identities

$$
\sinh \left(e_{j+2}\right) \sinh \left(e_{j-2}\right)=\cosh \left(e_{j}\right)
$$

so that the lengths of any two adjacent sides determine the lengths of the others; and there is a right-angled pentagon (unique up to isometry) whenever any (equivalently all) successive pairs of lengths satisfy the inequality

$$
\sinh \left(e_{j}\right) \sinh \left(e_{j+1}\right)>1 .
$$

If we make the substitution $X_{j}=\operatorname{coth}^{2}\left(\ell_{j}\right)$, the length identity (5) is equivalent to the cross-ratio identity (3), and the length inequality (6) is equivalent to the cross-ratio inequality (4).

Thus we can identify $\mathcal{P}$ with the space of cyclically ordered 5-tuples in $\mathbb{R} \mathbb{P}^{1}$, up to projective equivalence. If we identify $\mathbb{R} \mathbb{P}^{1}$ with the unit circle in $\mathbb{C}$, one normalization of the coordinates on this space that respects the symmetry (up to a rotation) is to set $p_{0}=1$ and insist that $\sum p_{j}=0$. This corresponds to viewing $\mathbb{R P}^{1}$ in the hyperbolic "visual metric" as seen from the barycenter of the $p_{j}$. Concatenating the vectors $p_{j}$ forms a convex equilateral Euclidean pentagon-i.e., one whose sides have a common length; thus we obtain a natural correspondence between convex equilateral Euclidean pentagons and right-angled hyperbolic pentagons.

If an equilateral Euclidean pentagon has internal angles $\alpha_{j}$, then any two successive angles determine the rest, by the formulae

$$
\cos \left(\alpha_{j}\right)+\cos \left(\alpha_{j+1}\right)-\cos \left(\alpha_{j}+\alpha_{j+1}\right)-\cos \left(\alpha_{j+3}\right)=1 / 2 .
$$

The parameter space is two-dimensional, and is compactified by adding edges, resp., vertices, corresponding to degenerations when one, resp., two (nonadjacent), angles go to $\pi$. This compactified parameter space $\mathcal{P}$ - the pentagon of equilateral Euclidean pentagons, or equivalently the pentagon of pentagons of pentagons-is depicted in Figure 5.

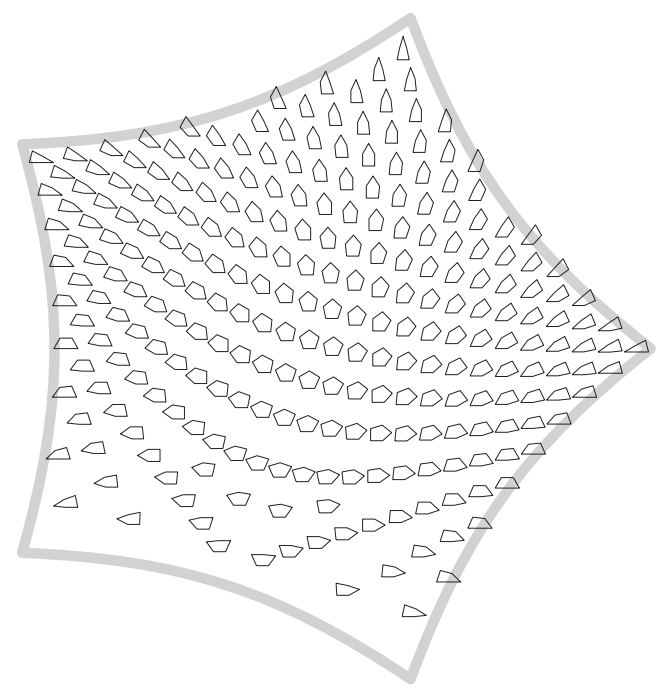

Figure 5. The pentagon of pentagons of pentagons.

That which is crooked cannot be made straight; and that which is wanting cannot be numbered.

AUTHOR'S NOTE. The author learned the essentials of this story from Problem 2.3.12 in Bill Thurston's book Three-Dimensional Geometry and Topology, Vol. 1, from Princeton University Press. Related matters are developed (considerably further) in Bill's famous paper "Shapes of polyhedra and triangulations of the sphere," Geom. Topol. Monogr. 1 (1998), 511-549.

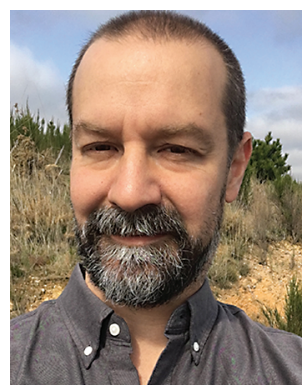

Danny Calegari

\section{Credits}

Figures and photo of Danny Calegari are courtesy of Danny Calegari. 